\title{
A novel mechanism of vitamin D anti-inflammatory/ antioxidative potential in type 2 diabetic patients on metformin therapy
}

\author{
Milena Cojic ${ }^{1}$, Radivoj Kocic ${ }^{2}$, Aleksandra Klisic ${ }^{1}$, Ljiljana Cvejanov-Kezunovic ${ }^{1}$, Nebojsa Kavaric ${ }^{1}$, \\ Gordana Kocic ${ }^{3}$
}

\begin{abstract}
${ }^{1}$ Primary Health Care Center, University of Montenegro, Faculty of Medicine, Podgorica, Montenegro

${ }^{2}$ Clinic for Endocrinology, Faculty of Medicine, University of Nis, Nis, Serbia

${ }^{3}$ Institute of Biochemistry, Faculty of Medicine, University of Nis, Nis, Serbia
\end{abstract}

Submitted: 2 October 2019

Accepted: 9 January 2020

Arch Med Sci 2020; 16 (5): 1004-1012

DOI: https://doi.org/10.5114/aoms.2020.92832

Copyright @ 2020 Termedia \& Banach

\section{Abstract}

Introduction: The performed study focused on determining the effect of vitamin D supplementation on enzymes involved in both inflammation and reactive oxygen species (ROS) production and ROS degradation in patients with type 2 diabetes mellitus (T2DM).

Material and methods: The 6-month follow-up, randomized, controlled study included 140 patients with T2DM, $\geq 30$ years old, with good metabolic control, treated with metformin and lifestyle advice only. All patients were randomly assigned to two groups (70 each). Patients from the first group (Intervention group) were assigned to receive vitamin D3 $50000 \mathrm{IU}$ or $14000 \mathrm{IU}$ regarding their vitamin D baseline levels. Patients from the second (Metformin) group continued to receive only metformin during the 6-month study period.

Results: After 6 months, the myeloperoxidase activity was significantly lower and gradually decreased in the Intervention group by about $40 \%$, compared to the baseline measurement $(p=0.015)$ and compared to the Metformin group ( $p=0.001)$. After 6 months, the xanthine oxidase (XO) activity decreased significantly in the Intervention group compared to the baseline and $3^{\text {rd }}$ month levels $(p<0.001)$. In the Metformin group there was also a significant decrease in XO after 6 months compared to baseline $(p<0.001)$ and the $3^{\text {rd }}$ month $(p=0.003)$. The catalase activity significantly increased within the Intervention group only when comparing the $3^{\text {rd }}$ and $6^{\text {th }}$ month $(p=0.027)$.

Conclusions: Our study showed that vitamin D may improve endothelial dysfunction in patients with T2DM on metformin therapy by influencing two important factors implicated in the pathogenesis of diabetic complications - ROS production and inflammation, which can additionally contribute to a stable metabolic control during metformin therapy.

Key words: xanthine oxidase, diabetes mellitus type 2, myeloperoxidase, vitamin D supplementation.

\section{Introduction}

Type 2 diabetes mellitus (T2DM) is a complex metabolic disorder characterized by insulin resistance (IR) in target tissues and a relative defi-

\author{
Corresponding author: \\ Milena Cojic \\ Primary Health \\ Care Center \\ Faculty of Medicine \\ University of Montenegro \\ Podgorica, Montenegro \\ E-mail: milenarovcanin@ \\ yahoo.com
}


ciency of insulin secretion from pancreatic $\beta$-cells [1]. Current evidence reveals an important role of inflammation and oxidative stress (OS) in T2DM development and progression. Furthermore, chronic hyperglycemia and IR promote a pro-oxidant environment and production of reactive oxygen species (ROS), while exhausting the endogenous antioxidative defense system [2, 3]. The consequent oxidative imbalance leads to further progression of the disease and development of micro- and macrovascular diabetic complications [4]. Therefore, strategies for preventing or reducing the inflammation and ROS imbalance could be a promising therapeutic tool in T2DM.

In recent decades, powerful evidence has shown that the physiological effects of vitamin $D$ are not limited only to bone metabolism. It is believed that vitamin $\mathrm{D}$ has an extensive range of non-calcemic roles, and the antioxidative effect is newly suggested but rarely studied $[2,5]$. Only a few controlled clinical trials have investigated the antioxidative effect of vitamin D supplementation in patients with T2DM, but a recent one confirmed that vitamin D can be used as a therapeutic agent along with other treatments for T2DM as it can improve glycated hemoglobin values $\left(\mathrm{HbA}_{1 c}\right)$ and IR. In the trials, different OS biomarkers were used, but most trials were focused on determining endogenous antioxidant status by assessing levels of defense enzymes (catalase, superoxide dismutase and glutathione peroxidase) and/or total antioxidant capacity. Some of the trials assessed OS by detecting products of oxidative damage, such as a lipid peroxidation marker (malondialdehyde), advanced glycation end products and advanced oxidation protein products. The data collected indicated that some OS outcomes were affected by the vitamin $D$ supplementation, while others were not. This may be owing to the heterogeneity of study protocols regarding dose, length and the manner in which vitamin D was administered as well as to the heterogeneity of OS markers considered [6, 7].

Additionally, none of the studies focused on determining the effect of vitamin D on enzymes involved in both inflammation and ROS production, such as myeloperoxidase (MPO), or ROS generation, such as xanthine oxidase $(\mathrm{XO})$, in patients with T2DM.

MPO is a multifunctional heme enzyme, serving as a typical immune defense enzyme against invading pathogens. It is localized in intracellular granules of neutrophils, monocytes and macrophages. As a marker of inflammatory response, it accounts for up to $5 \%$ of the total dry mass of mentioned inflammatory cells. It is responsible for the generation of bactericidal hypochlorous acid $(\mathrm{HOCl})$, which has a strong oxidative property during respiratory burst reactions although it is not a free radical from the chemical point of view $[8,9]$.

Chronic activation of MPO may lead to oxidative modification of a number of cellular and extracellular biomolecules, such as cell signaling proteins, cysteine-rich peptides, extracellular matrix proteins, lipoproteins, cholesterol, membrane phospholipids and nucleic acids, with consequent production of haloamines, chloramines and bromamines. Oxidative modification of intracellular and membrane components of vascular endothelial cells may lead to the development of endothelial dysfunction, vascular damage and atherosclerosis. A causal relationship was documented by the accumulation of MPO in atherosclerotic lesions [10-15].

Besides the oxidative burst generated by the immune and inflammatory cell defense system, the other source of vascular superoxide in the circulation is the XO catalytic reaction. Being a part of the dehydrogenase-oxidase inter-convertible system, this molybdenum iron-sulfur enzyme exhibits broad substrate specificity, but the main substrates are the purine bases hypoxanthine and xanthine. The final oxidation product is uric acid (2,6,8-trioxypurine), accompanied by the release of superoxide anion radical $\left(\mathrm{O}_{2}^{-}\right)$and hydrogen peroxide $\left(\mathrm{H}_{2} \mathrm{O}_{2}\right)[16,17]$.

In vascular endothelial cells, the enzyme may exist as cytoplasmic and membrane-bound XO. Capillary endothelial damage may liberate cytoplasmic enzyme, while the adhered immune cells may induce the release of membrane-bound XO. The oxidation of the sulfhydryl residues or limited proteolytic digestion may further contribute to production of $\mathrm{XO}$ on account of pre-existing xanthine dehydrogenase in ischemic conditions $[18,19]$.

A bidirectional relation between the two prooxidative enzymes XO and MPO is initiated on the one hand by XO-catalyzed liberation of ROS, which induce the release of pro-inflammatory cytokines, and activate neutrophils and macrophage cells, which consequently release MPO. On the other hand, MPO can use the XO-liberated product urate as a physiological substrate, producing urate radical, 5-hydroxyisourate and allantoin, which may exacerbate inflammatory tissue damage $[20,21]$.

The increased activity of both MPO and XO has been reported as a mediating risk factor for T2DM. The ROS produced by MPO and XO have been documented to be related to diabetic complications, especially to vascular remodeling, endothelial dysfunction, atherosclerosis and chronic diabetic vascular complications [22-24].

A chronic increase of ROS, presumably of $\mathrm{H}_{2} \mathrm{O}_{2}$, may induce oxidative damage of pancreatic $\beta$-cells, which in T2DM may further aggravate 
glucose homeostasis and metabolic control due to compromised insulin secretion. The first antioxidative defense enzyme capable of degrading $\mathrm{H}_{2} \mathrm{O}_{2}$ into oxygen and water is catalase. Clinical and experimental studies have confirmed a contributing role of reduced catalase activity in the development of T2DM, metabolic syndrome and insufficient pancreatic insulin secretion [25-28].

Taking into account the role of ROS and inflammation as interconnected players in atherosclerotic vascular complications of T2DM, we performed a study to test a possible anti-inflammatory and antioxidative effect of vitamin D supplementation through the activity of enzymes involved in ROS production (MPO and XO) and degradation (catalase) in patients with T2DM on therapy with metformin.

\section{Material and methods}

\section{Design and participants}

The study is a 6-month follow-up, randomized, controlled open-label investigation into the effects of vitamin D supplementation on glycemic control and OS markers. A total of 140 patients with T2DM were recruited from the Primary Health Care Center in Podgorica, Montenegro in a summer-winter period. The eligibility criteria stipulated men and women $\geq 30$ years old, who had good metabolic control $\left(\mathrm{HbA}_{1 \mathrm{c}} \leq 7 \%\right)$ and who were being treated with metformin and offered lifestyle advice only. The study excluded patients who used diabetes pharmacotherapy other than metformin, vitamin D supplements, drugs which affect vitamin D metabolism (corticosteroids and anticonvulsants) or if they had severe anemia, chronic liver or kidney failure, alcohol addiction, pregnancy; malabsorption; urolithiasis; hypercalcemia; body mass index $(B M I) \geq 40 \mathrm{~kg} / \mathrm{m}^{2}$ and any acute or chronic inflammatory condition. The study was conducted according to the Declaration of Helsin$\mathrm{ki}$ and Good clinical Practice guidelines. The study protocol was approved by the Ethical Committee of Primary Health Care Center in Podgorica and written informed consent was obtained from all participants.

\section{Intervention}

The participants were randomized to two groups (70 each) in the ratio $1: 1$. According to the US Endocrine society guidelines, the participants were considered vitamin $\mathrm{D}$ deficient if their 25(OH)D baseline levels were $\leq 50 \mathrm{nmol} / \mathrm{l}$. Suspected vitamin $D$ insufficiency was present if baseline levels were suboptimal, i.e. $\leq 75 \mathrm{nmol} / \mathrm{l}$ [29]. The participants from the first group (Intervention group) were prescribed one of two dosing regimens with cholecalciferol (vitamin $\mathrm{D}_{3}$ ), depend- ing on the baseline vitamin D levels. If they were vitamin $\mathrm{D}$ deficient, along with metformin they received $50000 \mathrm{IU}$ of vitamin $\mathrm{D}_{3}$ weekly (divided into equal daily doses) for 3 consecutive months to correct deficiency, followed by 2000 IU daily (14 000 IU weekly) for the next 3 months to maintain sufficient vitamin D levels. The participants from the same group whose vitamin $D$ baseline levels were $\geq 50 \mathrm{nmol} / \mathrm{l}$ received $2000 \mathrm{IU}$ of vitamin $D_{3}$ daily (14 000 IU weekly) along with metformin until the end of the study. Seventy participants from the second group (Metformin group) continued to receive only metformin During the 6-month study period.

The supplement adherence was carefully monitored. Twenty patients were excluded from the Intervention group since they did not adhere to the study protocol. In the Metformin group 2 patients were lost to follow-up (Figure 1).

\section{Follow-up and outcome measurement}

Anthropometric and laboratory assessments were performed at baseline (prior to dosing) and 3 and 6 months after the study. Waist circumference, body height, weight as well as systolic (SBP) and diastolic (DBP) blood pressure measurements were described in detail elsewhere [30].

Body mass index was calculated as weight ( $\mathrm{ki}$ lograms) divided by height (meters) squared.

Venous blood samples were collected after an overnight fast between 7:00 and 9:00 a.m. Serum levels of fasting blood glucose (FBG), ionized calcium $\left(\mathrm{Ca}^{++}\right)$, total cholesterol (TC), LDL cholesterol, HDL cholesterol and triglycerides (TG) were measured using standard enzymatic procedures (Roche Cobas 6000 c 501, Mannheim, Germany). The levels of $\mathrm{HbA}_{1 c}$ were determined immunoturbidimetrically (Roche Cobas 6000 c 501, Mannheim, Germany) using full blood with K2EDTA anticoagulants. Fasting insulin $(\mathrm{FI})$ and $25(\mathrm{OH}) \mathrm{D}$ serum levels were measured by commercial Roche tests based on the principle of electrochemiluminescence on the Cobas 6000/e601 automated analyzer (Roche Diagnostics, Mannheim, Germany).

Serum MPO activity was determined by a commercial ELISA test, using a pre-coated 96-well microplates with biotinylated target-specific antibodies. The sandwich assay is based on the reaction with the second (detector) antibody, when a substrate solution is added which reacted with the enzyme-antibody-target complex to produce coloring. The enzyme concentration was expressed as ng/l.

Serum XO activity was determined spectrophotometrically, based on the release of uric acid from xanthine as substrate [31], measured at $293 \mathrm{~nm}$, with slight modification in serum assay conditions [32]. The enzyme activity was expressed as U/I. 

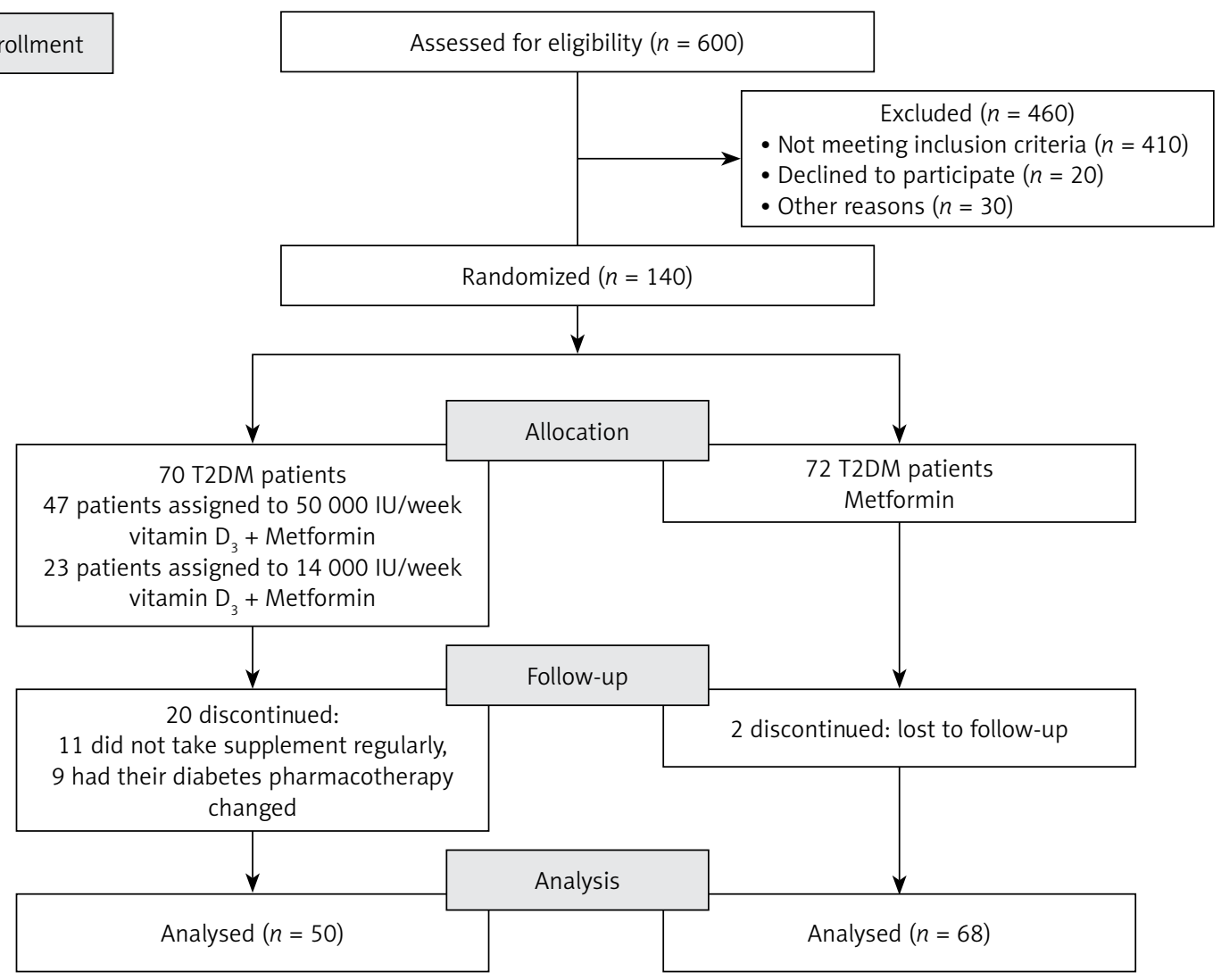

Figure 1. Participant flowchart and study design

Serum catalase activity was determined by using a spectrophotometric method, whereby the substrate hydrogen peroxide forms a stable yellow stained complex with molybdenum salts. The decrease in absorbance reflected the enzyme activity, expressed as Cat/l [33].

The homeostasis model assessment of insulin resistance (HOMA-IR) was calculated as formula: HOMA-IR = FBG $(\mathrm{mmol} / \mathrm{l}) \times \mathrm{FI}(\mu \mathrm{IU} / \mathrm{l}) / 22.5$ [34].

The triglyceride-glucose (TyG) index was calculated as the natural logarithm (In) of the product of FBG and TG using the formula: $\ln (\mathrm{TG}[\mathrm{mg} / \mathrm{dl}] \times$ fasting glucose [mg/dl]/2) [35].

\section{Statistical analysis}

The data are expressed as means \pm standard deviation (SD). For the comparison of the continuous variables between the groups Student's $t$ test was used if the distribution was normal. The Mann-Whitney test was used as a nonparametric test for data not normally distributed. Skewed continuous variables were natural log transformed before the analysis. The changes in the parameters by group were monitored over time using repeated ANOVA measures. Mean differences between the groups after 3 and after 6 months were assessed using linear regression analysis. All effects were adjusted for gender, baseline age and BMI. The significant $p$-value was set as 0.05 (two-sided).
Statistical procedures were performed using $R$ software (version 3.4.3) (R Foundation for Statistical Computing, Vienna, Austria) [36].

Sample size was calculated using the commercial statistical program $\mathrm{G}^{*}$ power for two-sided null hypothesis testing. The $F$-test or ANOVA for repeated measurements was used to determine the sample size in this software package. The estimated sample size was 49 patients per group considering type 1 error, $\alpha=0.05$ and statistical power $1-\beta=0.8$.

\section{Results}

Out of 140 participants, 118 successfully completed the study and reported no adverse effects as the result of the intervention. The baseline characteristics of the study population did not differ significantly between the groups. In the Intervention group the mean age of the patients was $60.0 \pm 8.0$ years and $55.9 \%$ were male. In the Metformin group the mean age was $64.4 \pm 8.1$ years and $42.0 \%$ were male.

About half of the participants in the Intervention and Metformin group had deficient baseline vitamin D levels ( $50 \%$ vs. $36.8 \%$ ). Suboptimal vitamin D status [25(OH)D $\leq 75 \mathrm{nmol} / \mathrm{l}]$ was observed in $84 \%$ participants in the Intervention group and in $76.4 \%$ in the Metformin group.

After 6 months of supplementation, vitamin D levels increased significantly in the Intervention 
group regarding baseline levels $(p=0.017)$. In the Metformin group vitamin D levels decreased significantly after 6 months $(p<0.001)$.

Change in $\mathrm{HbA}_{1 \mathrm{c}}$ levels was also significant within both groups. In the Intervention group there was a significant decrease in $\mathrm{HbA}_{1 \mathrm{c}}$ levels in the $3^{\text {rd }}$ month of supplementation $(p=0.023)$, and then a significant increase in the $6^{\text {th }}$ month compared to the $3^{\text {rd }}$ month $(p=0.005)$. In the Metformin group a similar pattern of change in $\mathrm{HbA}_{1 c}$ levels was observed but a significant difference was seen only between the $3^{\text {rd }}$ and $6^{\text {th }}$ month $(p<0.001)$.

HOMA-IR did not change significantly within the groups (Intervention group $p=0.458$, Metformin group $p=0.935$ ).

A significant difference, to the detriment of the Metformin group, over the 3- and 6-month follow-up period was observed in the: vitamin $D$ levels $(39.45, p<0.001 ; 36.22, p<0.001)$, BMI levels (2.83, $p=0.045 ; 6.61, p=0.005)$ and TyG index $(-0.33, p=0.003 ;-0.27, p=0.028)$ when adjusted for gender, baseline age and BMI (Table I).

Serum MPO was significantly reduced in the Intervention group over the period of study $(p=0.003)$. After 6 months, the MPO activity was significantly lower and gradually decreased, compared to the $3^{\text {rd }}$ month $(p=0.004)$ and about $40 \%$ compared to the baseline measurement ( $p=$ 0.015). In the Metformin group the MPO activity did not change significantly over time $(p=0.671)$ (Figure 2).

After 6 months, the XO activity decreased significantly in the Intervention group compared to the baseline and $3^{\text {rd }}$ month levels $(p<0.001)$ by more than $36 \%$. The same pattern was observed in the Metformin group, with a significant change between baseline and the $6^{\text {th }}$ month $(p<0.001)$ and between the $3^{\text {rd }}$ and $6^{\text {th }}$ month $(p=0.003)$ (Figure 3).

The catalase activity significantly decreased in the Intervention group after 3 months ( $p=0.010)$, but after 6 months of supplementation there was a significant increase compared to the $3^{\text {rd }}$ month $(p=0.027)$.

In the Metformin group the initial and final catalase activity did not differ significantly $(p=$ 0.809).

At the end of the study a significant difference between the groups regarding OS biomarkers was seen only in MPO levels $(p=0.001)$ (Table I).

\section{Discussion}

Our study showed that vitamin D supplementation in patients with T2DM resulted in a significant decrease in pro-inflammatory and pro-oxidant enzymes MPO and XO as well as a significant increase in antioxidative enzyme activity (as mea- sured with catalase activity). To our knowledge, none of the previous researchers investigated the effects of vitamin $D$ on both enzymes (XO and MPO) in patients with T2DM.

Our study showed a significant decrease of MPO within the Intervention group, but also a significant mean difference between the groups after 6 months $(p=0.001)$. Only one study evaluated the effect of vitamin D on MPO activity in T2DM patients [37]. Our results are partially in accordance with the study conducted by Nikooyeh et al., which demonstrated that the consumption of vitamin D fortified yogurt decreased MPO activity within the vitamin $D$ group, but failed to demonstrate a significant difference when compared to the group which consumed plain yogurt [37]. This may be due to the consumption of lower vitamin D doses (500 IU daily) compared to the doses in our study (7120 IU daily and/or 2000 IU daily).

It is well documented that MPO is a multifunctional enzyme, which may generate different ROS. The reaction is initiated by the assembly of one $\mathrm{NADPH}$ oxidase with plasma membrane, followed by the release of $\mathrm{O}_{2}{ }^{--}$, which may dismutate to $\mathrm{H}_{2} \mathrm{O}_{2}$. Finally, reactive $\mathrm{HOCl}$ is generated in the presence of $\mathrm{H}_{2} \mathrm{O}_{2}$ and the chloride ion. Generated $\mathrm{HOCl}$, judging by the oxidative properties, helps in destroying microbes and malignant cells [8, 38].

A number of the cell and extracellular biomolecules containing nitrogen can be a target for $\mathrm{HOCl}$ attack, when multiple types of chloramines are formed, presumably in reactions with histidine, lysine and arginine, purine and pyrimidine bases, nucleosides, nucleotides, DNA, RNA, amino sugars and glycosaminoglycans and amino alcohols of phospholipids [10, 39]. Susceptible amino acids in proteins are tyrosine and cysteine. MPO-induced oxidative modification of tyrosine generates halotyrosines, 3-chlorotyrosine, 3,5-dihalotyrosine and dityrosine $[12,40]$.

MPO is a pro-oxidant enzyme, also involved in pathogenesis of atherosclerosis as it modifies lipoprotein particles, particularly atherogenic LDL lipoprotein. In this way, both the apoprotein structure and lipid core of LDL cholesterol are subject to oxidative modification to a more atherogenic form, which can avoid LDL-receptor stimulated and controlled uptake and favor scavenger receptor LDL uncontrolled uptake followed by the formation of foam cells. The target chemical composition of oxidative damage is a double bond in the structure of unsaturated fatty acids and cholesterol in circulating lipoproteins and structural phospholipids and cholesterol localized on the cell membrane. In this way substrates, the unsaturated fatty acids and cholesterol form chlorohydrins and N,N-dichlorinated phospholipids, after 


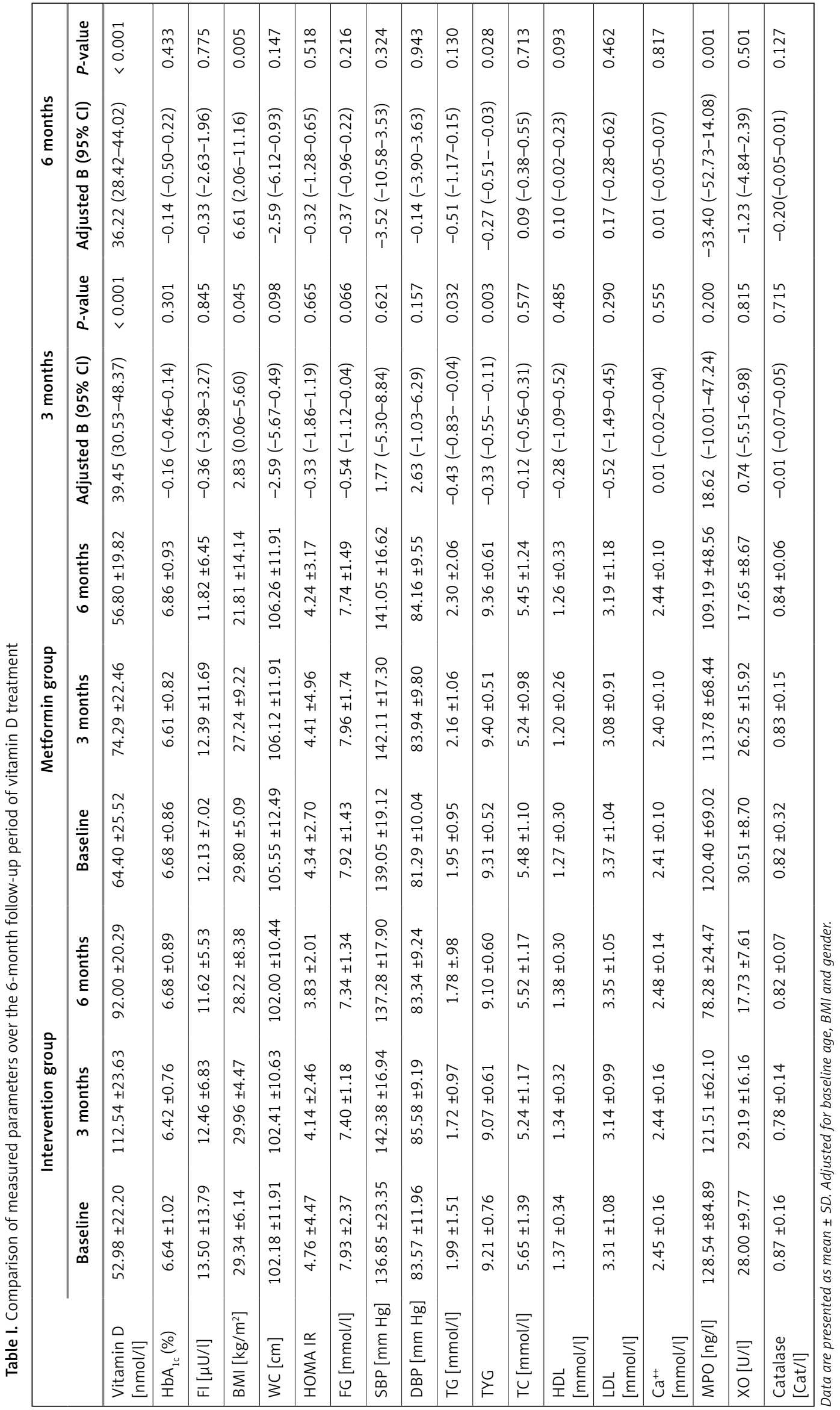




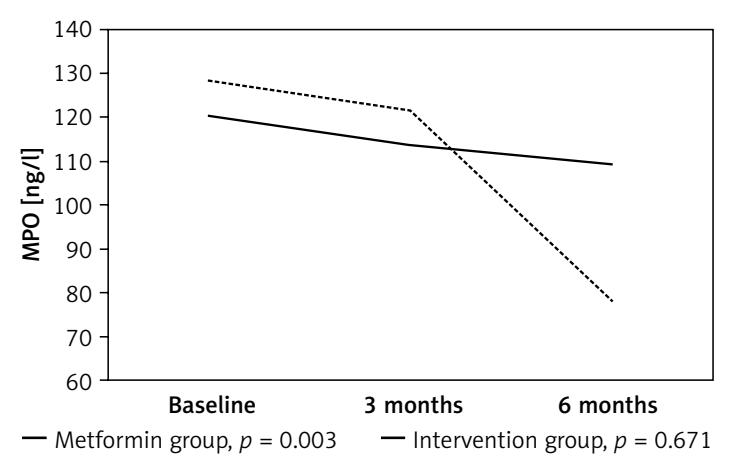

Figure 2. MPO activity during the study ( $p$-value on the graph - from repeated measure analysis) ${ }^{*} p=0.200$ for Intervention vs. Metformin group after 3 months: $p=0.001$ Intervention vs. Metformin group after 6 months)

exposure to $\mathrm{HOCl}$. The oxidative modification of HDL cholesterol forms a dysfunctional oxidized form [14, 15, 41-44]. Oxidatively modified tyrosine derivatives, together with MPO and activated macrophages, have been found in atherosclerotic lesions. Oxidatively modified cysteine may generate a cross-link with lysine or arginine, with the formation of sulfenic or sulfinic acid intermediates. Stimulated production of collagen by MPO may also contribute to vascular and extracellular matrix remodeling [13, 45-48].

The results obtained may be of importance in preventing the development and progression of cardiovascular diseases, by protecting coronary arteries from atherosclerotic and endothelial damage [49].

$\mathrm{XO}$ is an enzyme which plays an important role in purine catabolism by catalyzing the terminal oxidation of hypoxanthine to xanthine to uric acid [50]. A reaction catalyzed by $\mathrm{XO}$ produces free radicals, the superoxide anion radical and hydrogen peroxide. Along with uric acid, they have been implicated in several damaging pathways resulting in endothelial dysfunction in T2DM patients $[51,52]$. Although the literature regarding $\mathrm{XO}$ and T2DM association in humans is obscure, it has been proposed that the inhibition of XO activity leads to improved endothelial function in patients with T2DM and associated mild hypertension [53].

The results from our report showed a significant decrease in XO activity in the Intervention group after 6 months of supplementation with vitamin D. In the Metformin group there was also a significant decrease in $\mathrm{XO}$ activity after 6 months. A plausible explanation for the lack of significant mean difference between groups could be that our patients had good metabolic control, while hyperglycemia is one of the stimuli that trigger XO release from damage after inflammatory attack on vascular endothelial cells in T2DM patients

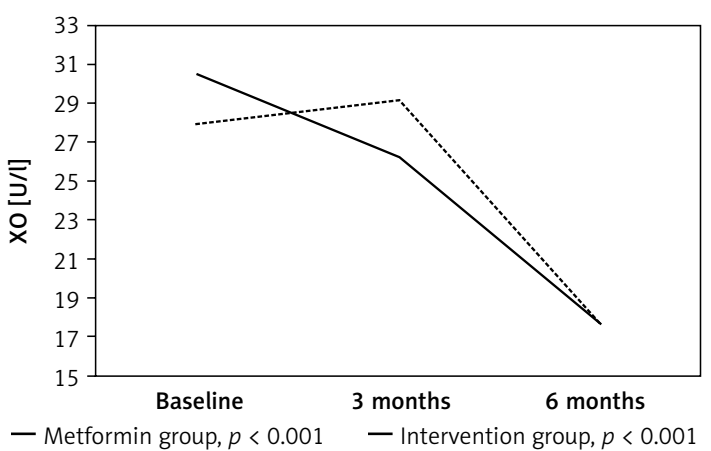

Figure 3. XO activity during the study ( $p$-value on the graph - from repeated measure analysis) ${ }^{*} p=0.815$ for Intervention vs. Metformin group after 3 months: $p=0.501$ Intervention vs. Metformin group after 6 months)

$[23,54]$. Other stimuli include hypoxia and hypercholesterolemia [50, 55]. Besides metabolic control, studies also reported some other confounders which can influence XO activity. Metformin could be one of them. Ćosić et al. found that metformin produced a significant reduction of XO activity, which was greater than the relatively small decrease in the concentration of glucose [56].

The antioxidant status in our patients was evaluated through catalase activity. This hemoprotein is the main regulator of $\mathrm{H}_{2} \mathrm{O}_{2}$ degradation [25]. It scavenges excessive $\mathrm{H}_{2} \mathrm{O}_{2}$ produced by $\mathrm{XO}$ and other prooxidants, preventing ROS-mediated cell damage in that way. Many studies have demonstrated diminished antioxidative defense in patients with T2DM [57]. According to the literature, vitamin D could enhance ROS removal by stimulating the activity of antioxidative enzymes. One of the proposed mechanisms is that vitamin $D$ regulates expression of genes involved in the control of several antioxidative and detoxifying enzymes [58, 59]. In accordance with our expectations, study data showed a significant increase in catalase activity after long-term supplementation in the Intervention group while there was no significant change in the Metformin group. Alatawi et al. also demonstrated that vitamin D and Ca supplementation resulted in a significant increase in catalase activity in streptozotocin-induced diabetic rats [60].

Vitamin D effects related to the ROS-inflammation axis have been proposed recently and have not been well studied so far. The data from experimental studies implied that vitamin D supplementation could have beneficial effects mainly through decreasing lipid peroxidation and improving antioxidative defense [61].

In conclusion, our study sheds light on a novel mechanism of both anti-inflammatory and antioxidative effects of vitamin $D$ through the influence on MPO, XO and catalase activity. In this way, vitamin $D$ could improve endothelial dysfunction 
in patients with T2DM on metformin therapy by influencing two important factors implicated in pathogenesis of diabetic complications - ROS production and inflammation, which can additionally contribute to stable metabolic control.

\section{Conflict of interest}

The authors declare no conflict of interest.

\section{References}

1. American Diabetes Association. Diagnosis and classification of diabetes mellitus. Diabetes Care 2009; 32 (Suppl 1): S62-7.

2. Nikooyeh B, Neyestani TR. Oxidative stress, type 2 diabetes and vitamin D: past, present and future. Diabetes Metab Res Rev 2016; 32: 260-7.

3. Rains JL, Jain SK. Oxidative stress, insulin signaling and diabetes. Free Radic Biol Med 2011; 50: 567-75.

4. Giacco F, Brownlee M. Oxidative stress and diabetic complications. Circ Res 2010; 107: 1058-70.

5. Mokhtari Z, Hekmatdoost A, Nourian M. Antioxidant efficacy of vitamin D. J Parathyr Dis 2017; 5: 11-6.

6. Tagliaferri S, Porri D, De Giuseppe R, Manuelli M, Alessio $F$, Cena $H$. The controversial role of vitamin $D$ as an antioxidant: results from randomised controlled trials. Nutr Res Rev 2019; 32: 99-105.

7. Hu Z, Chen J, Sun X, Wang L, Wang A. Efficacy of vitamin $D$ supplementation on glycemic control in type 2 diabetes patients: a meta-analysis of interventional studies. Medicine 2019; 98: e14970.

8. Simsek O, Kocael A, Kocael P, et al. Inflammatory mediators in the diagnosis and treatment of acute pancreatitis: pentraxin-3, procalcitonin and myeloperoxidase. Arch Med Sci 2018; 14: 288-96.

9. Kato Y. Neutrophil myeloperoxidase and its substrates: formation of specific markers and reactive compounds during inflammation. J Clin Biochem Nutr 2016; 58: 99-104.

10. Winterbourn CC, Kettle AJ. Biomarkers of myeloperoxidase-derived hypochlorous acid. Free Radic Biol Med 2000; 29: 403-9.

11. Kato Y, Ono S, Kitamoto N, Kettle AJ. Covalent modification of cytoskeletal proteins in neuronal cells by tryptamine-4,5-dione. Redox Biol 2014; 2C: 983-90.

12. Fu S, Wang H, Davies M, Dean R. Reactions of hypochlorous acid with tyrosine and peptidyl-tyrosyl residues give dichlorinated and aldehydic products in addition to 3-chlorotyrosine. J Biol Chem 2000; 275: 10851-8.

13. DeNichilo MO, Panagopoulos V, Rayner TE, Borowicz RA, Greenwood JE, Evdokiou A. Peroxidase enzymes regulate collagen extracellular matrix biosynthesis. Am J Pathol 2015; 185: 1372-84.

14. Mirmohammadsadeghi A, Gharipour M, Roohafza $H$, Dianatkhah M, Sadeghi M. Effects of selenium supplementation on paraoxonase-1 and myeloperoxidase activity in subjects with cardiovascular disease: the Selenegene study, a double-blind randomized controlled trial. Arch Med Sci Atheroscler Dis 2018; 3: e112-8.

15. Kawai Y, Kiyokawa H, Kimura Y, Kato Y, Tsuchiya K, Terao J. Hypochlorous acid-derived modification of phospholipids: characterization of aminophospholipids as regulatory molecules for lipid peroxidation. Biochemistry 2006; 45: 14201-11.

16. Pacher P, Nivorozhkin A, Szabo C. Therapeutic effects of xanthine oxidase inhibitors: renaissance half a century after the discovery of allopurinol. Pharmacol Rev 2006; 58: 87-114.

17. Yiginer O, Ozcelik F, Inanc T, et al. Allopurinol improves endothelial function and reduces oxidant-inflammatory enzyme of myeloperoxidase in metabolic syndrome. Clin Res Cardiol 2008; 97: 334-40.

18. Kisic B, Miric D, Zoric L, et al. Xanthine oxidase activity in patients with age-related cataract associated with hypertension. Braz J Med Biol Res 2018; 51: e6129.

19. Batteli MG, Polito L, Bolognesi A. Xanthine oxidoreductase in atherosclerosis pathogenesis: not only oxidative stress. Atherosclerosis 2014; 237: 562-7.

20. Van der Zwan LP, Scheffer PG, Dekker JM, Stehouwer CD, Heine RJ, Teerlink T. Hyperglycemia and oxidative stress strengthen the association between myeloperoxidase and blood pressure. Hypertension 2010; 55: 1366-72.

21. Meotti FC, Jameson GN, Turner R, et al. Urate as a physiological substrate for myeloperoxidase: implications for hyperuricemia and inflammation. J Biol Chem 2011; 286: 12901-11.

22. Wiersma JJ, Meuwese MC, van Miert JN, et al. Diabetes mellitus type 2 is associated with higher levels of myeloperoxidase. Med Sci Monit 2008; 14: CR406-10.

23. Azenabor A, Erivona R, Adejumo E, Ozuruoke D, Azenabor R. Xanthine oxidase activity in type 2 diabetic Nigerians. Diabetes Metab Syndr 2019; 13: 2021-4.

24. Gomez Garcia A, Rivera Rodriguez M, Gomez Alonso C, Rodriguez Ochoa DY, Alvarez Aguilar C. Myeloperoxidase is associated with insulin resistance and inflammation in overweight subjects with first-degree relatives with type 2 diabetes mellitus. Diabetes Metab J 2015; 39: 59-65.

25. Goth L. Catalase deficiency and type 2 diabetes. Diabetes Care 2008; 31: e93.

26. Goth L, Nagy T, Káplár M. Acatalasemia and type 2 diabetes mellitus. Orv Hetil 2015; 156: 393-8.

27. Verma S, Alam R, Ahmad I, Singla D, Ali K, Hussain ME. Effect of glycemic control and disease duration on cardiac autonomic function and oxidative stress in type 2 diabetes mellitus. J Diabetes Metab Disord 2018; 17: 149-58.

28. Siddiqui A, Desai NG, Sharma SB, Aslam M, Sinha UK, Madhu SV. Association of oxidative stress and inflammatory markers with chronic stress in patients with newly diagnosed type 2 diabetes. Diabetes Metab Res Rev 2019; 35: e3147.

29. Holick MF, Binkley NC, Bischoff-Ferrari HA, et al. Evaluation, treatment, and prevention of vitamin $D$ deficiency: an Endocrine Society clinical practice guideline. J Clin Endocrinol Metab 2011; 96: 1911-30.

30. Klisic A, Kotur-Stevuljevic J, Kavaric N, Martinovic M, Matic $M$. The association between follicle stimulating hormone and glutathione peroxidase activity is dependent on abdominal obesity in postmenopausal women. Eat Weight Disord 2018; 23: 133-41.

31. Kizaki H, Sakurada T. Simple micro-assay methods for enzymes of purine metabolism. J Lab Clin Med 1977; 89: 1135-44.

32. Boban M, Kocic G, Radenkovic S, et al. Circulating purine compounds, uric acid, and xanthine oxidase/dehydrogenase relationship in essential hypertension and end stage renal disease. Ren Fail 2014; 36: 613-8.

33. Goth L. Serum catalase: reversibly formed charge isoform of erythrocyte catalase. Clin Chem 1991; 37: 2043-7.

34. Klisic A, Kavaric N, Jovanovic M, Soldatovic I, Gligorovic-Barhanovic N, Kotur-Stevuljevic J. Bioavailable testosterone is independently associated with Fatty Liver Index in postmenopausal women. Arch Med Sci 2017; 13: 1188-96. 
35. Kang B, Yang Y, Lee EY, et al. Triglycerides/glucose index is a useful surrogate marker of insulin resistance among adolescents. Int J Obes 2017; 41: 789-92.

36. $\mathrm{R}$ Core Team: A language and environment for statistical computing. R Foundation for Statistical Computing, 2014; Vienna, Austria. URL http://www.R-project.org/.

37. Nikooyeh B, Neyestani TR, Tayebinejad N, et al. Daily intake of vitamin D- or calcium-vitamin D-fortified Persian yogurt drink (doogh) attenuates diabetes-induced oxidative stress: evidence for antioxidative properties of vitamin D. J Hum Nutr Diet 2014; 27: 276-83.

38. Pattison DI, Davies MJ. Reactions of myeloperoxidase-derived oxidants with biological substrates: gaining chemical insight into human inflammatory diseases. Curr Med Chem 2006; 13: 3271-90.

39. Rees MD, Hawkins CL, Davies MJ. Hypochlorite-mediated fragmentation of hyaluronan, chondritin sulfates, and related $\mathrm{N}$-acetyl glycosamines: evidence for chloramide intermediates, free radical transfer reactios, and site-specific fragmentation. J Am Chem Soc 2003; 125: 13719-33.

40. Pattison DI, Hawkins CL, Davies MJ. Hypochlorous acid-mediated protein oxidation: how important are chloramine transfer reactions and protein tertiary structure? Biochemistry 2007; 46: 9853-64.

41. Pattison DI, Hawkins CL, Davies MJ. Hypochlorous acid-mediated oxidation of lipid components and antioxidants present in low-density lipoproteins: absolute rate constants, product analysis and computational modeling. Chem Res Toxicol 2003; 16: 439-49.

42. Thukkani AK, McHowat J, Hsu FF, Brennan ML, Hazen SL, Ford DA. Identification of alpha-chloro fatty aldehydes and unsaturated lysophosphatidylcholine molecular species in human atherosclerotic lesions. Circulation 2003; 108: 3128-33.

43. Pattison DI, Hawkins CL, Davies MJ. What are the plasma targets of the oxidant hypochlorous acid? A kinetic modeling approach. Chem Res Toxicol 2009; 22: 807-17.

44. Shao B, Oda MN, Bergt C, et al. Myeloperoxidase impairs ABCA1-dependent cholesterol efflux through methionineoxidation and site-specific tyrosine chlorination of apolipoprotein A-I. J Biol Chem 2006; 281: 9001-4.

45. Carr AC, Winterbourn CC. Oxidation of neutrophil glutathione and protein thiols by myeloperoxidase-derived hypochlorous acid. Biochem J 1997; 327: 275-81.

46. Hawkins CL, Pattison DI, Davies MJ. Hypochlorite-induced oxidation of amino acids, peptides and proteins. Amino Acids 2003; 25: 259-74.

47. Fu X, Mueller DM, Heinecke JW. Generation of intramolecular and intermolecular sulfenamides, sulfinamides, and sulfonamides by hypochlorous acid: a potential pathway for oxidative cross-linking of low-density lipoprotein by myeloperoxidase. Biochemistry 2002; 41: 1293-301.

48. Pullar JM, Vissers MC, Winterbourn CC. Glutathione oxidation by hypochlorous acid in endothelial cells produces glutathione sulfonamide as a major product but not glutathione disulfide. J Biol Chem 2001; 276: 22120-5.

49. Nicholls SJ, Hazen SL. Myeloperoxidase and cardiovascular disease. Arterioscler Thromb Vasc Biol 2005; 25: 1102-11.

50. Münzel T, Gori T, Bruno RM, Taddei S. Is oxidative stress a therapeutic target in cardiovascular disease? Eur Heart J 2010; 31: 2741-8.

51. Battelli MG, Bortolotti M, Polito L, Bolognesi A. Metabolic syndrome and cancer risk: the role of xanthine oxidoreductase. Redox Biol 2019; 21: 101070.

52. Banerjee $M$, Vats P. Reactive metabolites and antioxidant gene polymorphisms in type 2 diabetes mellitus. Redox Biol 2014; 2: 170-7.
53. Butler R, Morris AD, Belch JJ, Hill A, Struthers AD. Allopurinol normalizes endothelial dysfunction in type 2 diabetics with mild hypertension. Hypertension 2000; 35: 746-51.

54. Desco MC, Asensi M, Márquez R, et al. Xanthine oxidase is involved in free radical production in type 1 diabetes: protection by allopurinol. Diabetes 2002; 51: 1118-24.

55. Sunagawa S, Shirakura T, Hokama N, et al. Activity of xanthine oxidase in plasma correlates with indices of insulin resistance and liver dysfunction in patients with type 2 diabetes mellitus and metabolic syndrome: a pilot exploratory study. J Diabetes Investig 2019; 10: 94-103.

56. Ćosić V, Antić S, Pesić M, Jovanović O, Kundalić S, Djordjević VB. Monotherapy with metformin: does it improve hypoxia in type 2 diabetic patients? Clin Chem Lab Med 2001; 39: 818-21.

57. Jandrić-Balen M, Božikov V, Božikov J, Metelko Ž, Jandrić I, Romić Ž. Impact of glycemic control on antioxidant enzyme activity in patients with type 2 diabetes mellitus. Diabetol Croat 2004; 33: 131-5.

58. Bhat $M$, Ismail A. Vitamin D treatment protects against and reverses oxidative stress induced muscle proteolysis. J Steroid Biochem Mol Biol 2015; 152: 171-9.

59. Sepidarkish M, Farsi F, Akbari-Fakhrabadi M, et al. The effect of vitamin D supplementation on oxidative stress parameters: a systematic review and meta-analysis of clinical trials. Pharmacol Res 2019; 139: 141-52.

60. Alatawi FS, Faridi UA, Alatawi MS. Effect of treatment with vitamin D plus calcium on oxidative stress in streptozotocin-induced diabetic rats. Saudi Pharm J 2018; 26: $1208-13$.

61. Neyestani TR. Vitamin D, oxidative stress and diabetes: is there a link? In: Diabetes: Oxidative Stress and Dietary Antioxidants. Academic Press 2014; 111-20. 